\title{
(De)Constructing Dimensions
}

\author{
Nima Arkani-Hamed, Andrew G. Cohen ${ }^{\dagger}$ and Howard Georgi ${ }^{\ddagger}$ \\ Lyman Laboratory of Physics \\ Harvard University \\ Cambridge, MA 02138
}

\begin{abstract}
We construct renormalizable, asymptotically free, four dimensional gauge theories that dynamically generate a fifth dimension.
\end{abstract}

*arkani@bose.harvard.edu

Permanent address: Department of Physics, UC Berkeley, Berkeley, CA 94720

$\dagger$ cohen@andy . bu . edu

Permanent address: Physics Department, Boston University, Boston, MA 02215

${ }_{\ddagger}^{\ddagger}$ georgi@physics . harvard.edu 


\section{Introduction}

The world is apparently four-dimensional. But it is possible that at distances shorter than those yet probed the Universe may best be described by a theory with more than the conventional one time and three space coordinates. A simple model of such extra dimensions is a theory of fields living on a spacetime with four extended dimensions, plus one or more additional compact dimensions. At distances large compared to the size of these compact dimensions, such a theory appears four dimensional: gauge forces fall off like the square of the distance, free energies of massless degrees of freedom scale like the fourth power of the temperature, etc. At energies corresponding to the inverse compactification size, Kaluza-Klein excitations appear with a spectrum dictated by the detailed nature of the compact space. At energies much higher than this scale, the extra dimensions become manifest: physics at distances small compared to the compactification size is insensitive to the compactification, and the theory appears higher dimensional.

Unfortunately these higher dimensional field theories have dimensionful couplings and therefore require a cut-off. As energies approach this cut-off, physics depends sensitively on the cut-off procedure, typically becoming strongly coupled. This makes it difficult to address what happens at energies above the cut-off. Indeed, quantum gravity in four dimensions is challenging for similar reasons. Nevertheless, a few UV completions of higher-dimensional field theories have been suggested, each realizing the higher-dimensional theory as the low energy limit of some more fundamental theory with a sensible high energy behavior. One possibility is that the cut-off of the higher dimensional field theory coincides with the fundamental Planck scale, where gravity also becomes strong. In itself this does not allow us to say anything about the behavior of the theory at energies above the cut-off since super-Planckian quantum gravity is poorly understood. Moreover, the UV difficulties with higher-dimensional field theories are unrelated to gravity, and it is therefore interesting to search for UV completions of higher-dimensional field theories where gravity is completely decoupled. Some examples of this kind have emerged in non-gravitational subsectors of superstring theory, including $(0,2)$ super-conformal theories, little string theories and open-membrane theories of various kinds [1, 2, for example]. Unfortunately these theories are strongly coupled and typically difficult to understand. Furthermore, they can not be defined in more than six dimensions, and seem to rely on unbroken supersymmetry in an essential way.

In this paper we describe a new way of UV completing higher-dimensional field theories. Instead of starting with extra dimensions, we build them. In an inversion of the usual picture, these models are four-dimensional at very high energies. They are renormalizable and in most cases even asymptotically free. Extra dimensions emerge dynamically at low energies, in a simple and calculable way. This allows us to study many mysterious features of higherdimensional field theories on a firm footing, without worrying about the unknown physics of the UV cutoff. Even more important, our construction of extra dimensions puts higherdimensional physics into a broader context, and serves as a departure point for exploring more radical and even more interesting new possibilities. 


\section{$2 \quad S U(n) \times S U(m)$ moose}

Our example field theories, all of which will be four dimensional, contain gauge fields and fermions, and are conveniently summarized in a pictorial representation, referred to variously as "moose" [3, for example] or "quiver" [4] diagrams. In such diagrams gauge groups are represented by open circles, and fermions by single directed lines attached to these circles. A line directed away from a circle corresponds to a set of Weyl fermions transforming as the fundamental representation of the gauge group, while a line directed toward a circle corresponds to a set of Weyl fermions transforming as the complex conjugate of the fundamental representation. The moose diagram we will consider is the $N$-sided polygon representing

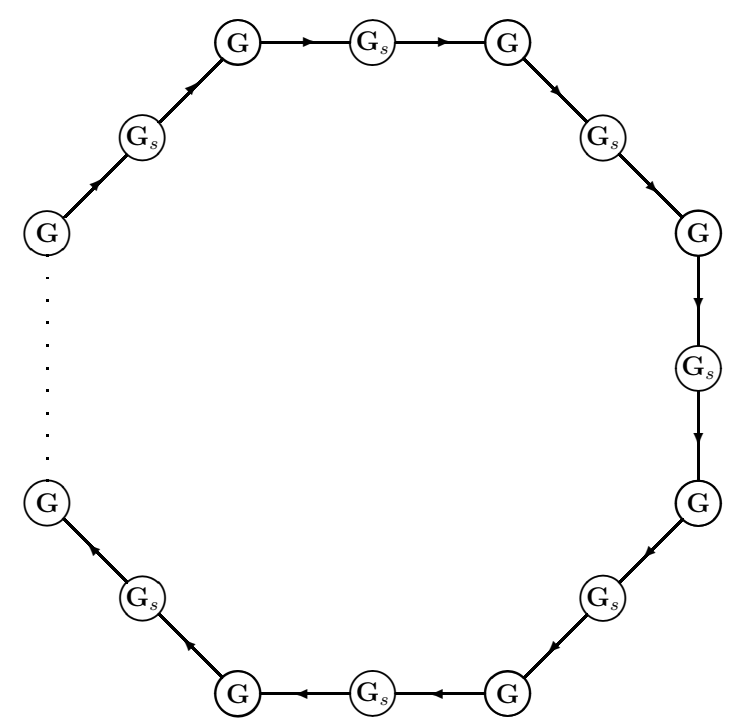

Figure 1: A moose diagram.

a field theory with a $\mathbf{G}^{N} \times \mathbf{G}_{s}^{N}$ gauge group and fermions transforming bi-linearly under "nearest-neighbor" pairs of gauge transformations.

For definiteness we will take $\mathbf{G}=S U(m)$ and $\mathbf{G}_{s}=S U(n)$. We will impose a cyclic symmetry to keep all $S U(m)$ gauge couplings equal to a common value $g$, and all $S U(n)$ gauge couplings equal to $g_{s}$. By dimensional transmutation we may equally well describe this theory by two corresponding dimensionful parameters, $\Lambda$ and $\Lambda_{s}$. Each side of this polygon describes two types of fermions transforming under the three gauge groups associated with this side, $S U_{i}(m) \times S U_{i}(n) \times S U_{i+1}(m)$ :

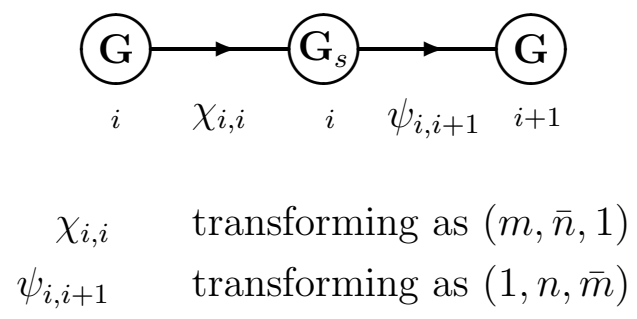

where $i=1, \ldots, N$ (and $i=0$ is periodically identified with $i=N)$. 
The field theory defined by this diagram is both anomaly and asymptotically free for a wide range of $m$ and $n$. At distances short compared to both $1 / \Lambda$ and $1 / \Lambda_{s}$, the theory is well-described by ( $N$ copies of) four-dimensional weakly interacting massless fermions and gauge bosons.

What does the theory look like at longer distances? In the limit where $\Lambda_{s} \gg \Lambda$ the long distance behavior is also simple. At energy scales near $\Lambda_{s}$ the $S U(m)$ gauge coupling is quite weak, and may be treated perturbatively. At this scale each of the $S U(n)$ groups become strong, causing the fermions to condense in pairs: a non-zero expectation value forms for each pair of fermions connected to a given strong gauge group:

$$
\left\langle\chi_{i, i} \psi_{i, i+1}\right\rangle \sim 4 \pi f_{s}^{3} U_{i, i+1} \quad i=1, \ldots, N
$$

where $f_{s} \sim \Lambda_{s} /(4 \pi)$ and $U_{i, i+1}$ is an $m \times m$ unitary matrix parameterizing the direction of the condensate. The confining strong interactions also produce a spectrum of "hadrons", analogues of ordinary glueballs and baryons, all with masses on the order of $\Lambda_{s} \sim 4 \pi f_{s}$. Below the scale $\Lambda_{s}$ the theory can be described as a $\Pi_{1}^{N} S U(m)$ gauge theory coupled to $N$ non-linear sigma model fields, each transforming as

$$
U_{i, i+1} \rightarrow \mathbf{g}_{i}^{-1}(x) U_{i, i+1} \mathbf{g}_{i+1}(x)
$$

We may use a diagram similar to the original moose to describe this "condensed" theory:

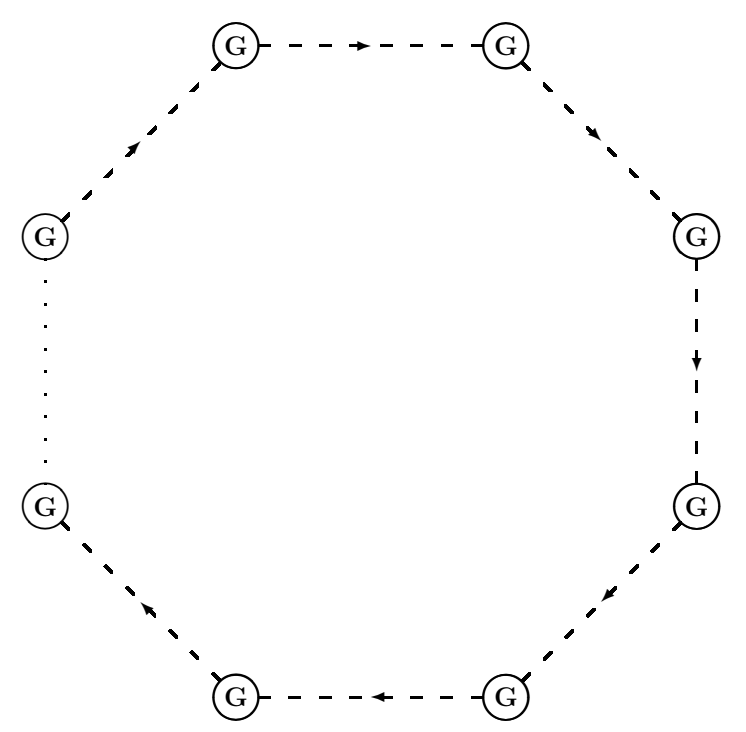

Figure 2: A condensed moose diagram

The low-energy effective action for this non-linear sigma model is

$$
S=\int d^{4} x\left(-\frac{1}{2 g^{2}} \sum_{j=1}^{N} \operatorname{tr} F_{j}^{2}+f_{s}^{2} \sum_{j=1}^{N} \operatorname{tr}\left[\left(D_{\mu} U_{j, j+1}\right)^{\dagger} D^{\mu} U_{j, j+1}\right]+\cdots\right)
$$

where the covariant derivative is $D_{\mu} U_{j, j+1} \equiv \partial_{\mu} U_{j, j+1}-i A_{\mu}^{j} U_{j, j+1}+i U_{j, j+1} A_{\mu}^{j+1}$ and the dots represent higher dimension operators that are irrelevant at low energies. The action for 
the non-linear sigma model fields connects the gauge fields at neighboring sites. In fact we recognize (2.5) as simply a discretized action for a five-dimensional gauge theory with gauge group $S U(m)$, where only the fifth dimension has been latticized. The non-linear sigma model fields are precisely the link variables of a lattice gauge theory, and the condensed moose diagram is a picture of the fifth dimension! It is remarkable that the moose diagram has transformed from a mnemonic for the particle content of a four-dimensional gauge theory to a new physical dimension of space at large distances.

The lattice spacing and the circumference of the fifth dimension are

$$
a=\frac{1}{g f_{s}}, \quad R=N a .
$$

The five-dimensional gauge coupling is

$$
\frac{1}{g_{5}^{2}}=\frac{1}{a g^{2}}=\frac{f_{s}}{g}
$$

We may eliminate any lingering doubt as to the five dimensional nature of this theory by calculating the spectrum of the $N$ gauge multiplets. The fluctuations of the condensates $U_{i, i+1}$ higgs the gauge group down to the diagonal subgroup. The gauge boson mass matrix is

$$
g^{2} f_{s}^{2}\left(\begin{array}{ccccccc}
2 & -1 & 0 & 0 & 0 & \ldots & -1 \\
-1 & 2 & -1 & 0 & 0 & \ldots & 0 \\
0 & -1 & 2 & -1 & 0 & \ldots & 0 \\
\vdots & \vdots & \ddots & \ddots & \ddots & \vdots & \vdots \\
0 & \ldots & 0 & 0 & -1 & 2 & -1 \\
-1 & \ldots & 0 & 0 & 0 & -1 & 2
\end{array}\right)
$$

This matrix is familiar from the physics of balls and springs, and its eigenvalues are easily calculated [5, for example], yielding a mass spectrum labeled by an integer $k$ satisfying $-N / 2<k \leq N / 2$

$$
M_{k}^{2}=4 g^{2} f_{s}^{2} \sin ^{2}\left(\frac{\pi k}{N}\right) \equiv\left(\frac{2}{a}\right)^{2} \sin ^{2}\left(\frac{p_{5} a}{2}\right),
$$

where $p_{5} \equiv 2 \pi k / R$ is the discrete five-dimensional momentum. The corresponding eigenvectors are of the form $\psi^{m} \sim \exp \left(i m p_{5} a\right)$. For $|k| \ll N / 2$ the masses become

$$
M_{k} \simeq\left|p_{k}\right|=\frac{2 \pi|k|}{R} \text {. }
$$

This is precisely the Kaluza-Klein spectrum for a five-dimensional gauge boson compactified on a circle of circumference $R$. The gauge coupling of the diagonal subgroup is $g_{4}^{2}=g^{2} / N$, and using (2.7) this gives

$$
\frac{1}{g_{4}^{2}}=\frac{R}{g_{5}^{2}}
$$

the usual relation between the five-dimensional and four-dimensional coupling constants. 


\section{What is a fifth dimension?}

For those familiar with lattice gauge theory, the appearance of the lattice action (2.5) makes clear that, in every sense, a true fifth dimension has appeared at large distances.' Nevertheless it is productive to examine how five-dimensional physics is reproduced.

The static potential between widely separated test charges is a common measure of the dimensionality of space: the potential is $r^{2-d}$ in $d$ spatial dimensions. Consider two charges at sites $\ell$ and $j$ separated by a distance $r$ in the ordinary three dimensions. The potential is a sum of Yukawa potentials from the KK modes, multiplied by the couplings to each mode

$$
V_{j \ell}(r) \sim \frac{g^{2}}{N} \sum_{k} e^{i(j-\ell) p_{k} a} \frac{e^{-M_{k} r}}{r}
$$

For $a \ll r \ll R$ the masses $M_{k}$ that contribute significantly to (3.1) can be accurately approximated by (2.10) and the sum in (3.1) can be approximated by an integral,

$$
V(r) \sim \frac{g^{2}}{N} \int_{-\infty}^{\infty} d k e^{2 \pi i k a(j-\ell) / R} \frac{e^{-2 \pi|k| r / R}}{r} \sim \frac{g_{5}^{2}}{a^{2}(j-\ell)^{2}+r^{2}}=\frac{g_{5}^{2}}{r_{5}^{2}+r^{2}}
$$

where $r_{5}=a(j-\ell)$ is the distance between the charges in the fifth dimension. The inversesquare fall-off is a sure sign of a fifth dimension, and is very different from the behavior of the potential at distances much shorter than $a$ where the theory looks four-dimensional. At distances much shorter than both $1 / \Lambda$ and $1 / \Lambda_{s}$, the potential between particles charged under any one of the gauge groups falls as $1 / r$ (modulo an additional slow variation due to the running of the gauge coupling). The contrast is even more striking for the potential between fermions charged under different gauge groups, corresponding to test charges separated in the fifth direction. At these short distances, this potential falls off much more rapidly: the leading interaction between different sites $(\ell, j)$ comes from a $2|\ell-j|+1$ loop diagram, and so falls off exponentially with $|\ell-j|$ as $g^{4|\ell-j|+4}$.

Every other physical measurement performed at distances much larger than $a$ but much smaller than $R$, will reveal a fifth dimension. Since the fifth dimension emerged dynamically, rather than being put in by hand, it is worthwhile to briefly address the question: what is a fifth dimension? Mathematically, any set of ordered points can be called a "dimension", but physically we need more. Particles should be able to move in the extra dimension; that is, they should carry labels, their co-ordinates in the fifth dimension, that change as they move in the fifth dimension. Furthermore, there should be a physical notion of locality in the extra dimension. This translates into the requirement of locality for the interactions in the theory. Particles with the same labels have the largest interaction, while particles with very different labels should interact only weakly.

These are the two defining properties of an extra dimension, and the fifth dimension we have generated possesses both of them. The gauge bosons propagate in the fifth dimension. Locality is a consequence of the nearest neighbor coupling structure of our moose, enforced by our choice of fermion content, gauge invariance and renormalizability. These

\footnotetext{
${ }^{1}$ Perhaps not every sense. We have not yet included gravitational interactions in our theory.
} 
constraints have another interesting consequence. There are infinitely many possible latticizations of a fifth-dimension, each with a different spectrum. We might then suspect that the spectrum (2.9) is easily modified. In fact, the particular lattice action (2.5) and the corresponding spectrum (2.9) followed uniquely from our renormalizable theory: the moose has made its choice.

\section{Lorentz invariance}

Extra dimensions may or may not be endowed with other properties as well. For instance they may be translationally invariant, or posses the full higher-dimensional Lorentz symmetry. Whether or not these additional properties arise in our constructions is a dynamical question. In the simple model we have presented, translational invariance is manifest, and the full $S O(4,1)$ Lorentz invariance also emerges at distances larger than $a$.

The lattice structure of the fifth dimension breaks five dimensional Lorentz invariance. For simplicity we will consider the limit $R \rightarrow \infty$, where the theory appears five-dimensional at arbitrarily long distances. In this limit $p_{5}$ becomes a continuous variable, and the dispersion relation for the five-dimensional gauge boson becomes

$$
E^{2}=\vec{p}^{2}+\left(\frac{2}{a}\right)^{2} \sin ^{2}\left(\frac{p_{5} a}{2}\right) \rightarrow \vec{p}^{2}+p_{5}^{2} \quad \text { as } a \rightarrow 0 .
$$

When $a \rightarrow 0$ the five dimensional Lorentz invariance is automatically restored. This might seem surprising because the fifth dimension is apparently quite different from the other three space dimensions. But in the limit of tiny spacing the only possible difference is a scale choice that we have eliminated to leading order in the weak coupling by defining the lattice spacing (2.6). Quantum effects will produce small changes in (2.6), but there is a definition of the lattice spacing that produces a Lorentz invariant limit in the full quantum theory. The fact that Lorentz invariance is automatic in this continuum limit is a consequence of the simplicity of this construction. In more complicated models, five-dimensional Lorentz invariance in the continuum limit may require tuning of parameters.

The violations of five dimensional Lorentz invariance due to the finite lattice size $a$ appear as a sequence of higher dimension operators in the five dimensional theory suppressed by powers of $p_{5} a$. We expect contributions of this size in any five dimensional theory because the inverse lattice spacing $1 / a$ plays the role of a cut-off. The difference here, compared to a standard five dimensional effective theory, is that the high energy theory above the cut-off scale is well defined, but lacks five dimensional Lorentz invariance. Thus the interactions suppressed by powers of the cut-off are calculable, but some break the Lorentz symmetry. Above the cut-off scale, there is no vestige of five dimensional Lorentz symmetry remaining, because the theory is perfectly four-dimensional at short distances.

\section{Phase structure}

The field theory associated with the diagram in figure 1 has an obvious symmetry between $\mathbf{G}$ and $\mathbf{G}_{s}$. It is clear that the discussion above for $\Lambda_{s} \gg \Lambda$ can be repeated for $\Lambda \gg \Lambda_{s}$. In 
this dual situation, the physics is described by the dual of the condensed moose in figure 2 . The condensates in this case are

$$
\left\langle\psi_{i, i+1} \chi_{i+1, i+1}\right\rangle \sim 4 \pi f^{3} V_{i, i+1} \quad i=1, \ldots, N .
$$

Again the physics is five dimensionful for $a \ll r \ll R$, but it is a different fifth dimension, dynamically generated by a different set of interactions and with a different set of gauge bosons.

The transition from (2.3) to (5.1) is theoretically fascinating, but somewhat puzzling, and we will not discuss it in detail here. But it is important to understand the approach to the transition because it bears on the possibly phenomenologically relevant question of how large the five dimensional gauge coupling can be. For example the heaviest of the KK modes has a mass of order $g\left(\Lambda_{s}\right) f_{s}$, parametrically lighter than the scale where $\mathbf{G}_{s}$ gets strong, $\Lambda_{s}$. How similar can we make these scales? Can we increase $g$ to the region of strong coupling as well?

For simplicity, let us take $N$ to infinity so that the physics appears five-dimensional at arbitrarily long distances. What happens as we change the ratio of $\Lambda$ to $\Lambda_{s}$ ? For $\Lambda \ll \Lambda_{s}$, where the analysis of section 2 applies, the residual gauge interactions at distances large compared to $a$ are very weak. In the five dimensional language, this is obvious because the gauge coupling is dimensional, $g_{5}^{2}=g^{2} a$, and its effects at distances of order $\ell$ are suppressed by powers of $g_{5}^{2} / \ell$. In the four dimensional language, one might worry that there is something wrong with this argument at distances large compared to $1 / \Lambda$, but such worry is groundless. The weak gauge group is higgsed by the condensate (2.3) down to a residual gauge group with coupling of order $g^{2} / N$ and thus becomes arbitrarily weak as we take $N \rightarrow \infty$.

What happens as we increase $\Lambda / \Lambda_{s}$ ? The gauge coupling $g_{5}^{2}=g^{2} a$ increases, but its effects remain tiny at large distances. We know that at some point as $\Lambda \rightarrow \Lambda_{s}$, an ecological disaster will occur, dramatically changing the nature of the long distance physics. But it is reasonable to suppose that the cataclysm will happen abruptly at some point $\Lambda \approx \Lambda_{s}$, where both gauge couplings are strong. The only signal at large distances of impending doom is that as $\Lambda \rightarrow \Lambda_{s}, g_{5}^{2}=g^{2} a$ gets large compared to $a$. This signals the imminent breakdown of the effective theory because dimensional couplings in an effective theory must not be large compared to the appropriate power of the cut-off. Even though the tree level interactions are still weak at long distances, the theory is losing control of its quantum corrections, a warning that anarchy is about to be loosed upon the world.

\section{Other completions}

The fifth dimension has appeared in the condensed moose because the non-linear sigma model fields allow the gauge field to "hop" from one site to the next. Since we could have obtained this directly as a latticization of the five-dimensional gauge theory, we might ask why we need the original moose model at all. The reason is that latticization in the fifth dimension does not cut-off divergences from large four-momenta: the four-dimensional non-linear sigma model of (2.5) is non-renormalizable, becoming strongly coupled at a scale $\sim 4 \pi f_{s}$. That is, this theory requires a UV completion. But this is familiar problem, with familiar solutions. The moose model we have constructed provides a UV completion in the same way that QCD 
completes the theory of pions. However purely perturbative completions are also possible. For example we could replace the non-linear sigma model with a renormalizable, linear sigma model; we replace each unitary field $U_{i, i+1}$ with a charged scalar field $\phi_{i, i+1}$. The action for this sigma-model will include a quartic potential for these scalars. If this potential produces vacuum expectation values for all the scalars at a scale $f_{s}$, this model is indistinguishable from our moose model at low energies. A fifth dimension then appears just as before.

In the linear sigma-moose, it is easy to include other degrees of freedom at the sites. Including appropriate couplings to the link variables will allow these fields to hop in the extra dimension as well. For example Yukawa couplings to fermions at the sites will produce hopping. Since the strength of this hopping term is unrelated to the gauge coupling, the fermions and gauge bosons propagate with different maximal speeds in the extra dimension. The resulting theory is five-dimensional, but without five-dimensional Lorentz invariance, even at large distances, although we can always tune the couplings to recover Lorentz invariance at long distance.

Although the linear sigma model example is renormalizable, the natural value for the vacuum expectation values of the scalar fields is the UV cut-off of the four-dimensional theory. We can avoid this standard problem of fundamental scalars in a standard perturbative way: by using supersymmetry. For simplicity we consider an $N=1, \mathbf{G}=S U(2)$ SUSY gauge version of our condensed moose, although extensions to larger gauge groups are straightforward. The arrows in this case are meaningless because the fundamental and anti-fundamental of $S U(2)$ are the same. The line connecting $i$ to $i+1$ denotes a bi-fundamental chiral superfield $\phi_{i}$, that we can think of as a $2 \times 2$ matrix. In addition to the gauge interactions, the theory has a superpotential

$$
W=\lambda \sum_{i} S_{i}\left(\operatorname{det} \phi_{i}-\mu^{2}\right)
$$

where $S_{i}$ are gauge singlet chiral fields, and $\mu$ is a mass scale. The theory is asymptotically free as long as the ratio $\lambda / g$ is not too large. The superpotential forces spontaneous symmetry breaking. Writing $\phi_{i}=\left(\mu+A_{i}\right) \exp \left(\sum_{i}^{a} \sigma^{a}\right)$, the superpotential pairs up $A_{i}$ and $S_{i}$ with a mass $\sim \lambda \mu$, while the $\Sigma_{i}$ contain the massless Goldstone bosons (together with their superpartners). At low energies we are left with a latticization of the five-dimensional $N=1$ $S U(2)$ gauge theory. In components, we have $S U(2)$ gauge bosons, together with a Dirac fermion and a real scalar in the adjoint representation. Note that in this theory no tuning of parameters is required to obtain five-dimensional Lorentz invariance. Supersymmetry guarantees that the gauge bosons, fermions and scalars propagate in the fifth-dimension with the same maximum velocity, and full five-dimensional Lorentz invariance is recovered at long distances.

\section{Conclusions and speculations}

We have constructed a fifth dimension dynamically in a four-dimensional renormalizable gauge theory. At long distances the physics is that of a compactified five-dimensional gauge theory with dimensionful couplings, that by itself would be non-renormalizable. This construction is easily extended to produce several extra dimensions. 
We can now investigate higher-dimensional physics in a well-defined setting. Questions involving energies higher than the naïve five-dimensional cut-off are straightforward in this context. Higher-dimensional phenomena, such as power-law running, localization of gauge fields and chiral fermions, orbifold compactification and supersymmetry breaking, to name a few, have straightforward constructions in our formalism [6].

The insight provided by our technique can work in both directions. Just as constructing extra dimensions in a renormalizable setting illuminates higher-dimensional physics, so too the physics of extra dimensions may suggest new phenomena in the context of purely four dimensional models that have no extra-dimensional interpretation. This has led to a novel approach for stabilizing the electroweak scale [7].

How does gravity fit in? The simplest possibility is to add four-dimensional gravity to our four-dimensional field theories. While the non-gravitational physics appears fivedimensional, gravity remains purely four-dimensional. Constructing extra dimensions in this way frees us from many of the naïve constraints of higher-dimensional model building. In particular the absence of gravity in the fifth-dimension eliminates many of the defects of non-standard gravity at high energies. For example radius stabilization is no longer an issue - there is no dynamical radius to stabilize! Rather the size of the extra dimension is set by the fixed parameters of the four-dimensional theory. As another example, the cosmology of extra dimensions is often troublesome. But in our construction, the Universe at temperatures above the naïve five-dimensional cut-off is described by a completely standard four-dimensional FRW cosmology. Without gravity the shape of the extra dimensions is not constrained by Einstein's equations. In fact the extra dimensions we have constructed may not have any simple manifold interpretation at all (consider a "figure-8")! It is also interesting to attempt to generate full five-dimensional gravity through a similar mechanism. This requires degrees of freedom that link the four-dimensional geometry at each site.

It is tempting to imagine that some or all of the three ordinary spatial dimensions may be generated dynamically. There is no obstacle in principle to constructing moose models in $2+1$ dimensions that generate a fourth dimension for non-gravitational fields. However a mechanism for obtaining four-dimensional gravity is essential.

The dynamical generation of extra dimensions within four-dimensional field theories allows exploration of higher-dimensional physics in a familiar context. Conversely insights from extra dimensions may be applied directly to purely four-dimensional models. Our construction serves as a link from extra dimensions to a new world of ideas.

\section{Acknowledgements}

H.G. is supported in part by the National Science Foundation under grant number NSFPHY/98-02709. A.G.C. is supported in part by the Department of Energy under grant number \#DE-FG02-91ER-40676. N.A-H. is supported in part by the Department of Energy. under Contracts DE-AC03-76SF00098, the National Science Foundation under grant PHY95-14797, the Alfred P. Sloan foundation, and the David and Lucille Packard Foundation.

\section{References}


[1] N. Seiberg, "Notes on theories with 16 supercharges," Nucl. Phys. Proc. Suppl. 67 (1998) 158-171, hep-th/9705117.

[2] R. Gopakumar, S. Minwalla, N. Seiberg, and A. Strominger, "OM theory in diverse dimensions," JHEP 08 (2000) 008, hep-th/0006062.

[3] H. Georgi, "A tool kit for builders of composite models," Nucl. Phys. B266 (1986) 274.

[4] M. R. Douglas and G. Moore, "D-branes, Quivers, and ALE Instantons," hep-th/9603167.

[5] H. Georgi, The Physics of Waves. Prentice-Hall, 1992.

[6] N. Arkani-Hamed, A. G. Cohen, and H. Georgi. In preparation.

[7] N. Arkani-Hamed, A. G. Cohen, and H. Georgi. In preparation. 